\title{
The Effects of miR-195-5p/MMP14 on Proliferation and Invasion of Cervical Carcinoma Cells Through TNF Signaling Pathway Based on Bioinformatics Analysis of Microarray Profiling
}

\author{
Min Lia Chun-Xia Ren ${ }^{a} \quad$ Jian-Mei Zhang ${ }^{a} \quad$ Xiao-Yan Xin ${ }^{b} \quad$ Teng Hua ${ }^{b}$ \\ Hong-Bin Wang ${ }^{c}$ Hong-Bo Wang ${ }^{b}$ \\ aDepartment of Gynecology, Xuhui Hospital Zhongshan Hospital, Fudan University, Shanghai Clinical \\ Center of the Chinese Academy of Science, Xuhui District Center Hospital of Shanghai, Shanghai, \\ bepartment of Obstetrics and Gynecology, Union Hospital, Tongji Medical College, Huazhong \\ University of Science and Technology, Wuhan, 'South Branch of the Six People's Hospital Affiliated to \\ Shanghai Jiao Tong University, Shanghai, China
}

\section{Key Words}

Cervical carcinoma $\cdot$ MiR-195-5p • MMP14 $・$ TNF signaling pathway

\begin{abstract}
Background/Aims: This study is aimed at identification of miR-195-5p/MMP14 expression in cervical cancer (CC) and their roles on cell proliferation and invasion profile of CC cells through TNF signaling pathway in CC. Methods: Microarray analysis, gene set enrichment analysis (GSEA) and DAVID were used to analyze differentially expressed miRNAs, mRNAs and signaling pathways. MiR-195-5p and MMP14 expression levels in CC cell were determined by qRT-PCR. Western blot was employed to measure MMP14 and TNF signaling pathwayrelating protein level. Luciferase reporter system was used to confirm the targeting relationship between MMP14 and miR-195-5p. Cell proliferation and invasion was respectively deeded by CCK8, transwell. In vivo experiment was carried out to study the impact of MMP14 and miR-195-5p on CC development in mice. Results: The microarray analysis and the results of qRT-PCR determined that miR-195-5p was under-expressed and MMP14 was over-expressed in CC cells. GSEA and DAVID analysis showed that TNF signaling pathway was regulated by miR-195-5p/MMP14 and activated in cervical carcinoma cells. The miR-195-5p and MMP14 have a negative regulation relation. In vivo experiment found that down-regulated MMP14
\end{abstract}

M. Li and C. Ren contributed equally to this work.

Hong-Bin Wang

and Hong-Bo Wang
South Branch of the Six People's Hospital Affiliated to Shanghai Jiao Tong University

No. 6600 Nanfeng Road, Fengxian District, Shanghai 201400 (China)

E-Mail huanghz52@126.com; Im621113@163.com 


\section{Cellular Physiology Cell Physiol Biochem 2018;50:1398-1413 \begin{tabular}{ll|l} 
DOl: 10.1159/000494602 & $\begin{array}{l}\text { O } 2018 \text { The Author(s). Published by S. Karger AG, Basel } \\
\text { www.karger.com/cpb }\end{array}$ \\
\cline { 2 - 4 }
\end{tabular} \\ Li et al.: miR-195-5p/MMP14 Suppresses the Progression of Cervical Carcinoma}

and up-regulated miR-195-5p suppressed the tumor development. Conclusion: Our results suggest that MMP14 is a direct target of miR-195-5p, and down-regulated MMP14 and upregulated miR-195-5p suppressed proliferation and invasion of CC cells by inhibiting TNF signaling pathway.

(C) 2018 The Author(s)

Published by S. Karger AG, Basel

\section{Introduction}

With a high incidence, cervical cancer (CC) remains the third most prevalent gynecological cancer diagnosed in females. Over 50 million new patients are diagnosed with CC yearly around the world, especially in developing countries, which account for over $80 \%$ of all worldwide cases [1-3]. Conventionally, radiotherapy and chemotherapy individually or both have been used for CC treatment. However, the curative effect for CC patients with chemo-resistance remains poor [4]. Therefore, it is of great significance to find alternative therapeutic methods for the treatment of CC.

MicroRNAs (miRNA/miR) are small non-coding RNAs with 18-25 nucleotides. They are reported to regulate protein expressions by binding to specific genes and inhibiting mRNA or protein translation process $[2,5]$. Numerous researches have demonstrated that miRNA expression level is closely related to human cancer progression and metastasis. Previous studies have shown that various miRNAs are abnormally expressed in CC tissues, and miR195 is one of them $[3,6]$. Some studies have been done to elucidate the role of miR-195 in CC cells, and it is believed that miR-195 functions as a tumor suppressing miRNA in CC [2]. In our study, we further investigated the function of miR-195-5p in CC and explicated the hidden mechanism of their relationship.

Matrix metalloproteinases 14 (MMP14), also known as membrane type 1 matrix metalloproteinase (MT1-MMP), is a member of MT-MMP subfamily. MMPs are a class of zincbinding proteases which lead to tumor metastasis by degrading extracellular matrix (ECM). Among them, MMP14 is reported as a tumor promoter by inhibiting cell adhesion molecules and some cytokines including pro-tumor necrosis factor- $\alpha$ (TNF- $\alpha$ ) [7]. A previous study has suggested that MMP14 is involved in CC development [8]. And a positive correlation between MMP14 and CC progression have been confirmed in previous studies, which claimed that MMP14 could promote angiogenes, lymph node metastasis and vascular invasion and that MMP14 overexpression predicted a poor prognosis of CC patients $[9,10]$. Several researchers explored the relationship between miRNAs and MMP14 in cancers, and found that miRNAs regulates tumorigenesis by modulating MMP14 [11]. However, the link between miR-195-5p and MMP14 in CC has not been explicated yet.

Tumor necrosis factor (TNF) is characterized by its anti-tumor performance in cancer cells. As a member of TNF superfamily of cytokines, TNF mediates cell processes such as inflammation, differentiation, proliferation and apoptosis [12]. More specifically, it has been proved that TNF has certain correlation with CC [1]. A previous study has suggested that MMP14 silencing results in a better treatment of patients resistant to anti-TNF therapy [13]. And TNF expression level is also mediated by miR-195 [14, 15]. Nevertheless, the underlying mechanisms are to be clarified. TNF receptor 1 (TNFR1), one of the activated receptors of TNF, contributes to the majority of TNF-induced events as a death receptor [12]. Additionally, TNF- $\alpha$ can effectively induce cell apoptosis by binding to TNFR1, and fatty acid synthase (FAS) is also recognized as a cytoplasmic "death domain" [16]. Therefore, in this study, we measured the protein expression profile of TNFR1, TNF- $\alpha$ and FAS to verify the activity of TNF signaling pathway.

The present study is aimed at expounding the behind relationship of miR-195-50p and MMP14 and finding their functions by regulating TNF signaling pathway in CC. 


\section{Cellular Physiology Cell Physiol Biochem 2018;50:1398-1413 \begin{tabular}{ll|l} 
and BiOChemistry & $\begin{array}{l}\text { DOI: 10.1159/000494602 } \\
\text { Published online: 25 October 2018 }\end{array}$ & $\begin{array}{l}\text { ( } 2018 \text { The Author(s). Published by S. Karger AG, Basel } \\
\text { www.karger.com/cpb }\end{array}$ \\
\hline
\end{tabular}}

Li et al.: miR-195-5p/MMP14 Suppresses the Progression of Cervical Carcinoma

\section{Materials and Methods}

\section{Bioinformatics analysis}

The data for analysis were collected from GSE9750 and GSE86100, GEO Database. The aberrantly expressed mRNAs and miRNAs were analyzed by $R$ and $R$ studio software. The aberrantly expressed signaling pathways were confirmed using the Kyoto Encyclopedia of Genes and Genomes (KEGG) and Gene ontology (GO) through Gene Set Enrichment Analysis (GSEA) and the online website DAVID Bioinformatics Resources 6.8 (http://david.abcc.ncifcrf.gov/). The interactive relationship between miR-195-5P and TNF signaling pathway was verified by DIANA website (http://snf-515788.vm.okeanos.grnet.gr/index.php?r=mirpath/ index). The bioinformatics prediction program we developed based on TargetScan (http://www.targetscan. org/vert_71/) and Mirnada (http://34.236.212.39/microrna/home.do) showed the binding sites that miR195-5p potentially with MMP14.

\section{Tissue samples}

Human CC tissues were obtained at Union Hospital, Tongji Medical College, Huazhong University of Science and Technology (Wuhan, China). Totally 36 paired CC tissues and non-cancerous adjacent tissues (NATs) were collected from CC patients post-primary surgery. All the patients had not undergone chemotherapy, radiotherapy or other treatment before surgery. CC tissues and NATs were preserved at $-80^{\circ} \mathrm{C}$ in liquid nitrogen immediately after extraction. Research Ethics Committee of Union Hospital, Tongji Medical College, Huazhong University of Science and Technology knew and approved of the present study. Informed consent was obtained from every patient involved in written form.

\section{Cell culture}

HeLa cells were obtained from the Union Hospital, Tongji Medical College, Huazhong University of Science and Technology (Wuhan, China) and maintained in Dulbecco's modified Eagle's medium (DMEM; Invitrogen Life Technologies, Carlsbad, CA, USA) containing 10\% fetal bovine serum (FBS; Invitrogen Life Technologies). All cells were incubated in a $100 \%$ humid atmosphere with $5 \% \mathrm{CO}_{2}$ at $37^{\circ} \mathrm{C}$.

\section{Cell transfection}

Cells were incubated in seven groups: Negative control (NC), si-MMP14, miR-195-5p-mimics, miR195-5p-inhibitor, si-TNF- $\alpha$ and si-MMP14 + miR-195-5p-inhibitor. NC, miR-195 mimics, miR-195 inhibitor, si-TNF- $\alpha$ and si-MMP14 were compounded by Shanghai GenePharma Co., Ltd. (Shanghai, China). Cells in the exponential phase of growth were seeded in a 6-well plate and maintained in DMEM without antibiotics. The transfection was carried out using Lipofectamine ${ }^{T M} 2000$ (Invitrogen; Thermo Fisher Scientific, Inc.) following the manufacturer's directions when the cell density reached $50-60 \%$.

\section{qRT-PCR}

TRIzol reagent (Invitrogen; Thermo Fisher Scientific, Inc.) was employed for total RNA (tRNA) isolation. PrimeScript ${ }^{\mathrm{TM}}$ RT Reagent kit (Takara Biotechnology Co., Ltd., Dalian China) was used for cDNA synthesis using $1 \mu \mathrm{g}$ tRNA. QRT-PCR was carried out using SYBR Premix Ex Taq Master mix (Takara Biotechnology Co, Ltd.) in an Applied Biosystems 7500 Real-

Time PCR system (Thermo Fisher Scientific, Inc.) in accordance with the manufacturer's directions. U6 small nuclear RNA (U6) and GAPDH were used as internal controls for miR-195-5p and MMP14 mRNA expression, respectively. The involved primers were present in Table 1. The data were analyzed using $2^{-\Delta \Delta c t}$ method. All experiments were performed for three times.
Table 1. Sequences for RT-PCR

\begin{tabular}{lc}
\hline cDNA & Sequences $\left(5^{\prime}-3^{\prime}\right)$ \\
\hline MiR-195-5p forward & ACACTCCAGCTGGGTAGCAGCACAGAAAT \\
MiR-195-5p reverse & TGGTGTCGTGGAGTCG \\
U6 forward & CTCGCTTCGGCAGCACA \\
U6 forward & AACGCTTCACGAATTTGCGT \\
MMP14 forward & TCGGCCCAAAGCAGCTTC \\
MMP14 reverse & CTTCATGGTGTCTGCATCAGC \\
GAPDH forward & TCAAGGCTGAGAACGGGAAG \\
GAPDH reverse & TGGACTCCACGACGTACTCA \\
\hline
\end{tabular}




\section{Cellular Physiology Cell Physiol Biochem 2018;50:1398-1413 \begin{tabular}{l|l|l} 
and Biochemistry Published OnIIne: 25 October 2018 & $\begin{array}{l}\text { (c) } 2018 \text { The Author(s). Published by S. Karger AG, Basel } \\
\text { www.karger.com/cpb }\end{array}$ \\
\hline
\end{tabular}}

Li et al.: miR-195-5p/MMP14 Suppresses the Progression of Cervical Carcinoma

\section{Western Blot}

The cells were washed twice using phosphate-buffered saline and lysed directly in lysis buffer (1 mmol/l PMSF, 1 mmol/l EDTA, 40 mmol/l Tris-HCl, $100 \mathrm{mmol} / \mathrm{l} \mathrm{NaVO3,} 150 \mathrm{mmol} / \mathrm{l} \mathrm{KCl}$ and 1\% Triton $\mathrm{X}-100)$ on ice for $15 \mathrm{~min}$. Subsequent to centrifugation $\left(12,000 \times \mathrm{g}\right.$ for $20 \mathrm{~min}$ at $4^{\circ} \mathrm{C}$ ), the Bradford protein assay kit (Bio-Rad Laboratories, Inc., Hercules, CA, USA) was used to determine the protein concentrations of the lysates. Equal amounts of proteins were isolated on 10\% SDS-PAGE gels and then electro-transferred onto a polyvinylidene difluoride membrane. $5 \%$ skimmed milk-Tris-buffered saline plus Tween-20 solution was used for the block of membranes and then the cells were incubated with anti-MMP14 antibody (1:5000; ab51074) or anti-beta actin antibody (1:1000; ab8226), anti-TNF Receptor I antibody (1:1000; ab19139), anti-TNF alpha antibody (1:20000; ab6671) and anti-Fas antibody (1:1000; ab82419). Following incubation with peroxidase-conjugated AffiniPure goat anti-rabbit IgG $(1: 2,000)$, Goat Anti-Mouse IgG H\&L (Alexa Fluor® 790) (1:10000), an HRP-conjugated goat anti-rabbit IgG polyclonal (1:5000), a Biotin-conjugated Goat anti-rabbit IgG polyclonal (1/2000), alexa-Fluor 488 conjugated donkey anti-rabbit polyclonal $(1: 1000)$.

\section{Dual Luciferase Reporter Gene assay}

HeLa cells were seeded into a 12-well plate. Transfection was conducted when the cells achieved $90 \%$ confluence with Lipofectamine ${ }^{\mathrm{TM}} 2000$ (Invitrogen, Carlsbad, CA, USA). HeLa cells were transfected with miR-195-5p or NC, and pGL3-MMP14-3'-UTR wild-type (wt) or pGL3-MMP14-3'-UTR-Mutation (mut). Activities of firefly and Renilla luciferase were measured using the Dual-Luciferase Reporter assay system (Promega Corporation, Madison, WI, USA) after the cells were preserved at $37^{\circ} \mathrm{C}$ for $48 \mathrm{~h}$ in accordance with the manufacturer's protocol. The activities of firefly luciferase were used as a control. All experiments were performed in triplicate.

\section{CCK-8 assay}

Cells were seeded at 2000 cell/well in 96-well plates for incubation after transfection. Cell proliferation was detected using a CCK-8 kit (Dojindo Laboratories) at the same time of each day in a week. Each assay was repeated at least three times.

\section{Transwell Invasion assay}

Matrigel invasion assay was performed using a 24-well transwell plates (costar) with polycarbonate filters (pore size, $8 \mu \mathrm{m}$ ). Matrigel was attenuated to $1 \mathrm{mg} / \mathrm{ml}$ with $4^{\circ} \mathrm{C}$ serum-free medium on the ice. $100 \mu \mathrm{l}$ attenuated matrigel was added to the upper chamber of the plates. Transwell was washed with serumfree medium for 3 times and then cell suspension with $2 \times 10^{5}$ cell $/ \mathrm{ml}$ was obtained. Next, matrigel was rinsed using serum-free medium and $100 \mu \mathrm{l}$ cell suspension was added to the upper chamber. For the lower chamber, $600 \mu \mathrm{l}$ medium with $20 \%$ FBS was added. After $20-24 \mathrm{~h}$ incubation in a $37^{\circ} \mathrm{C}$ incubator, the transwell was washed with PBS twice and then the cells were fixed using 5\% glutaraldehyde. Crystal violet $(0.1 \%)$ was used for staining after the cells were rinsed in PBS twice. The transwell was cultured at room temperature for $0.5 \mathrm{~h}$ and then washed in PBS twice. Transwell was finally observed under a microscope.

\section{Xenograft models established in nude mice}

The HeLa cells were stably transfected with miR-195-5p-mimics, control, or miR-195-5p-inhibitor. The three group cells were respectively subcutaneously injected into the 6 week-old nude mice (4 nude mice in each group, 2 males and 2 females) in the flank. Mouse weight and tumor size were measured biweekly. Tumor volumes were computed as follows: length $\times$ width $^{2} \times 1 / 2$. The tumor weights were measured 5 weeks after implantation and xenograft tumors tissues were collected for the following researches.

\section{Statistical analysis}

Differentially expressed miRNAs and mRNAs were screened by $\mid$ Fold change $\left(\log _{2} \mathrm{FC}\right) \mid>2$ and $P<0.0$. The functional annotation tool of GSEA and DAVID Bioinformatics Resources 6.8 were used to verify the remarkable enrichment of genes and pathways in resulting dataset. Significant pathways were identified using $\log _{10} \mathrm{P}$-value cut-off of 2. GraphPad Prism software 6.0 (San Diego, CA, USA) was chosen for data analysis. Three or more groups were compared using $t$-test. The results were presented as mean \pm SD (standard deviation). $P<0.05$ was considered statistically significant.

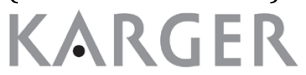




\section{Results}

MiR-195-5p was low expression and MMP14 was high expression in CC cells

The differentially expressed mRNAs in normal cervical epithelium and primary tumors were detected by mRNA microarray assay. To identify potential diagnostic and prognostic biomarkers, the ten most over expressed (GABBR1, CXCL13, MMP14, MMP1, HELLS, NEK2, SYNGR3, CDKN2A, SYCP2 and SPP1) and under expressed (KRT4, CRISP3, CRNN, MAL, ALOX12, KRT, CWH43, SLURP1, DSG1 and ENDOU) mRNAs in CC compared to the normal cervical epithelium that showed statistically significant differences in expression were

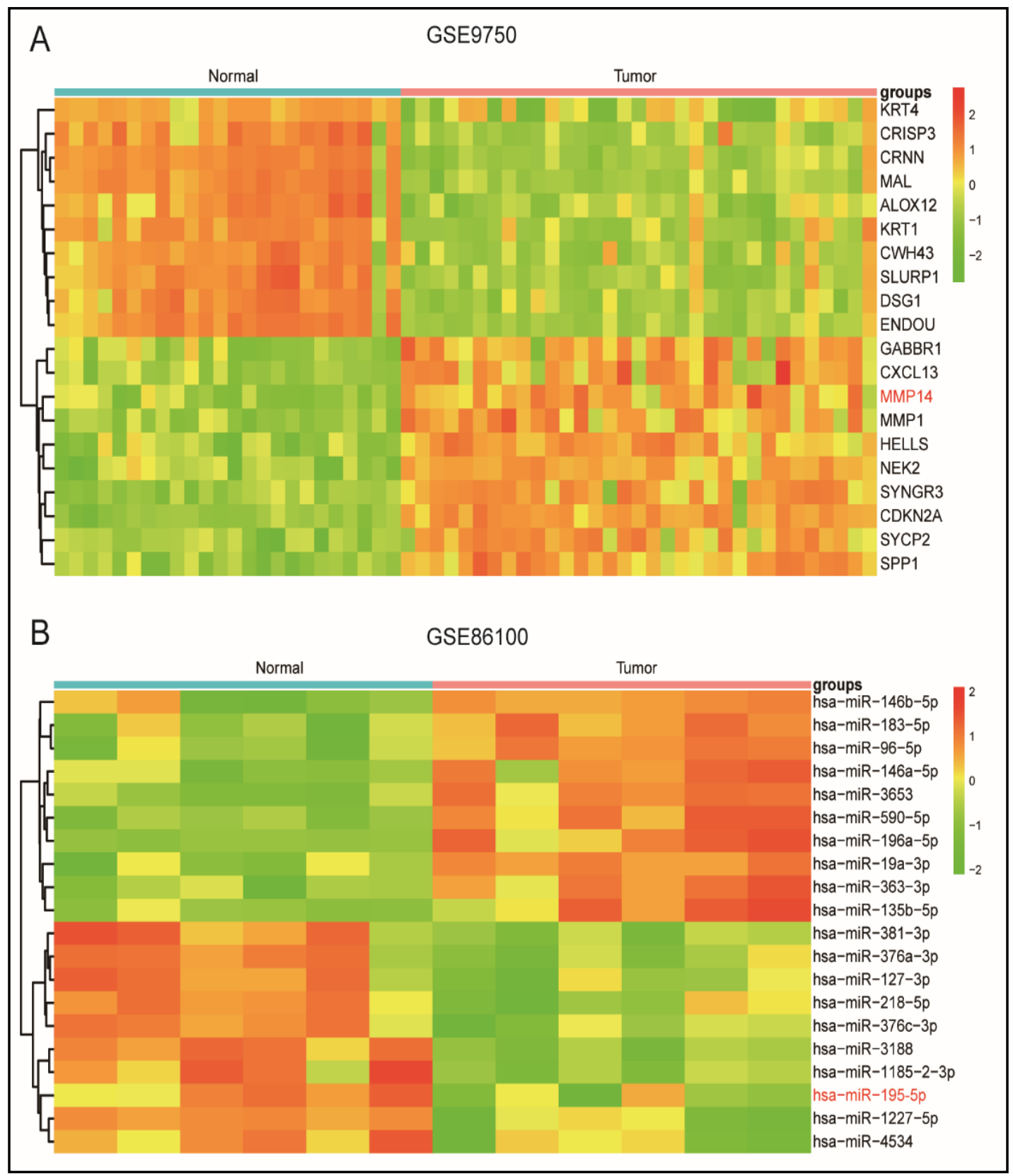

Fig. 1. MiR-195-5p was low expression and MMP14 was high expression in CC cells. (A) Heat map showed top 10 over expressive genes and top 10 down expressive genes in mRNAs microarray assay. (B) Heat map showed top 10 over expressive miRNAs and top 10 down expressive miRNAs in miRNAs microarray assay.

\section{KARGER}


selected in the heat map (Fig. 1A). Furthermore, the miRNA microarray assay displayed the differentially expressed miRNAs, the ten most over expressed (miR-146b-5p, -183-5p, -96-5p, -146a-5p, -3653, -590-5p, -196a-5p, -19a-3p, -363-3p and -135b-5p) and under expressed (miR-381-3p, 376a-3p, -127-3p, -218-5p, -376c-3p, -3188, -1185-2-3p, -195-5p and -1227-5p) (Fig. 1B).

\section{Potential functions of dysregulated mRNAs on mRNA microarray}

The aberrantly expressed mRNAs were used for the functional annotation analysis. After gene ontology function enrichment analysis, we obtained up-regulated function in normal which were listed on left and up-regulated function in tumor which were listed on right. Fig. 2A listed the highest 7 GO_ALL significant functions respectively in normal and tumor tissues. In addition, the gene ontology biological process (GO_BP) were listed by Fig. 2B, the gene ontology cellular component (GO_CC) were listed by Fig. 2C and the gene ontology molecular function (GO_MF) were listed by Fig. 2D.

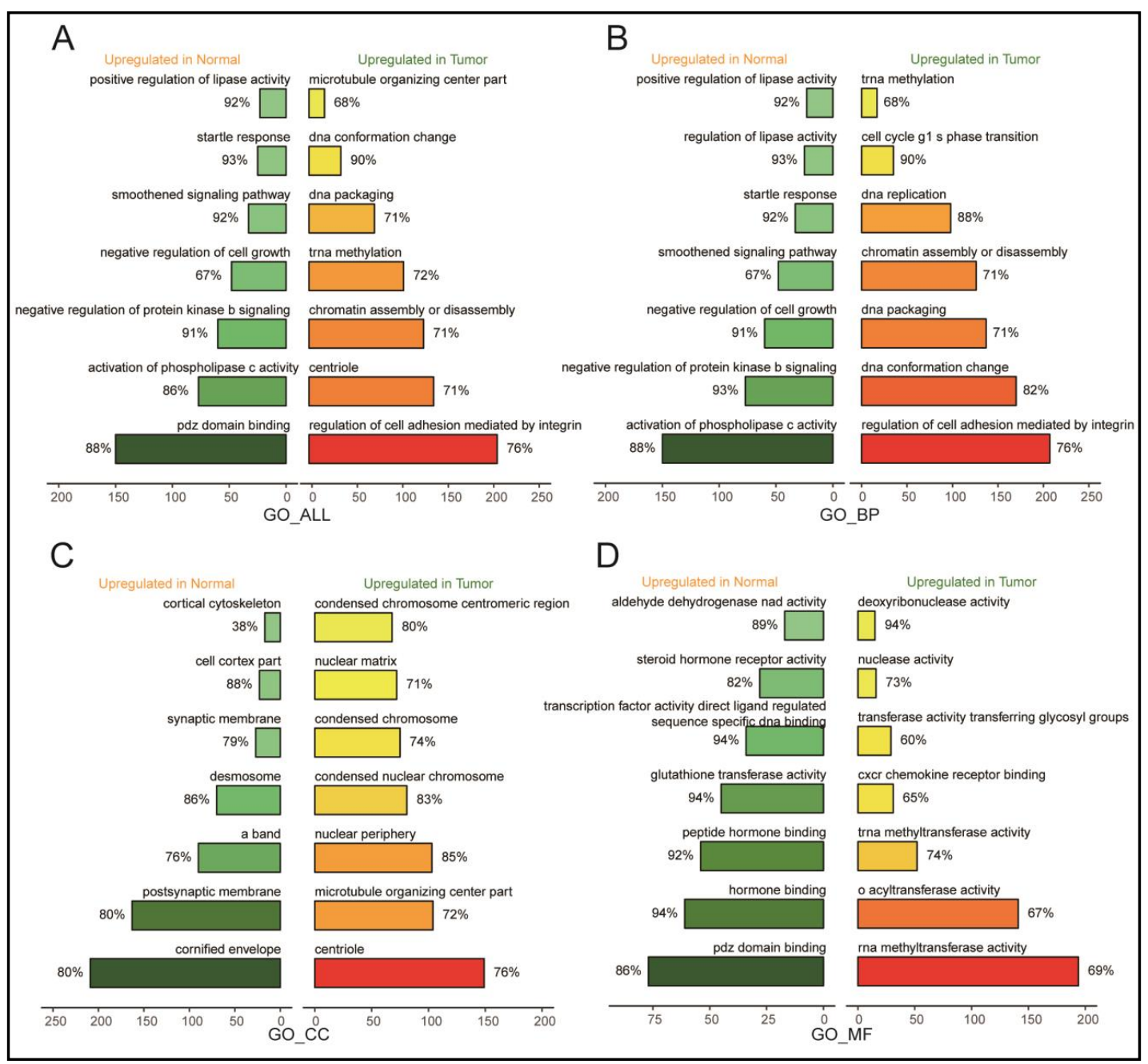

Fig. 2. Potential functions of dysregulated mRNAs on mRNA microarray. (A) The highest 7 GO_ALL significant functions respectively in normal and tumor tissues. (B) The highest 7 GO_BP significant functions respectively in normal and tumor tissues. (C) The highest 7 GO_CC significant functions respectively in normal and tumor tissues. (D) The highest 7 GO_MF significant functions respectively in normal and tumor tissues. 


\section{Cellular Physiology Cell Physiol Biochem 2018;50:1398-1413 \begin{tabular}{ll|l} 
DOl: 10.1159/000494602 & $\begin{array}{l}\text { O } 2018 \text { The Author(s). Published by S. Karger AG, Basel } \\
\text { wwww.karger.com/cpb }\end{array}$
\end{tabular} \\ Li et al.: miR-195-5p/MMP14 Suppresses the Progression of Cervical Carcinoma}

The TNF signaling pathway that activated in tumor tissues was the key pathway in progression of CC

The KEGG pathway enrichment analysis showed that the TNF signaling pathway was upregulated in tumor (Fig. 3A), which was verified by functional annotation analysis in DAVID (Fig. 3B). The venn diagram showed the result more intuitive, cell cycle and TNF signaling pathway were the common pathways that upregulated in tumor detected by KEGG pathway enrichment analysis and DAVID functional annotation analysis (Fig. 3C). Through the dot plot (Fig. 3D) and joy plot (Fig. 3E), we can received that the TNF signaling pathway was obviously activated in tumor with $P<0.05$. The Fig. 3F showed the TNF signaling pathway was exit in the 13 corporate significantly different pathways revealed by dot plot and joy plot.

The miR-195-5p can target the MMP14, and act on the TNF signaling pathway in CC

The result of STRING analysis indicated that MMP14 was the interactive gene of the TNF signaling pathway (Fig. 4A). GSEA analysis displayed that the TNF signaling pathway was activated in tumors (Fig. 4B). The differentially expressed mRNAs on the TNF signaling pathway was showed by heat map which detected that the MMP14 was over-expressed in tumor tissues (Fig. 4C). Furthermore, in order to exhibit the interactive relationship of miRNAs and mRNAs, we do the network plot which showed that miR-195-5p was low expression and MMP14 was high expression and the expression of them was negative correlation (Fig. 4D). We obtained the heat map which showed the interaction of the miR195-5p with the TNF signaling pathway by DIANA website. In order to show the important role of miR-195-5p in the cervical carcinoma more clearly, we made the venn diagram which showed hsa-miR-497-5p, hsa-miR-205-5p, hsa-miR-96-5p, hsa-miR-195-5p and hsa-miR218-5p were in the intersection of miRNAs associated with TNF signaling pathway which obtained by reverse searching TNF signal pathway on DIANA website, targeting miRNAs of the MMP14 and the miRNAs showed in the network (Fig. 4E-4F). The Fig. 4G obtained by TargetScan which suggested that miR-195-5p directly target MMP14. Taken together, we certified that miR-195-5p/MMP14 may be a key target for disease treatment.

In tissue experiment, miR-195-5p was low expression and MMP14 was high expression in CC tissues

To identify the functions of miR-195-5p in CC, miR-195 expression in CC tissues and NATs was measured by qRT-PCR and the result showed that miR-195 was considerably down-regulated in CC tissues compared with NATs $(P<0.05$; Fig. 5A). The results of qRT-PCR and western blot revealed that MMP14 mRNA and protein levels were both up-regulated in CC tissues compared with NATs $(P<0.05$; Fig. 5B-5C).

MMP14 was miR-195-5p's target gene not TNF- $\alpha$ or TNFR-1

Bioinformatics analysis showed that miR-195-5p directly targets MMP14 gene, an important regulator involved in tumor progression (Fig. 6A). For thoroughly verifying their relationship, we therefore constructed luciferase reporter vector containing 3'-UTR of MMP14. The reporter assay showed that miR-195-5p is able to markedly inhibit luciferase expression, whereas mutation of 6 nucleotides in 3'-UTR of MMP14 results in complete abrogation the suppressive effect (Fig. 6B).

MiR-195-5p influenced proliferation and invasion of the CC cells by regulating MMP14 through positive feedback to TNF signaling pathway

CCK-8 assay results indicated that the cells transfected with miR-195-5p-inhibitor had the maximum cell proliferation ability, the cells transfected in NC group and si-MMP14 + miR-195-5p-inhibitor group took the second place, and the cells in si-MMP14 or miR-195$5 p$-mimics group had the minimum cell proliferation ability $(P<0.05$, Fig. 7A). The level of MMP14 mRNA was detected by qRT-PCR showed that MMP14 was over-expressed in miR-195-5p-inhibitor group and under-expressed in si-MMP14 group and miR-195-5p- 


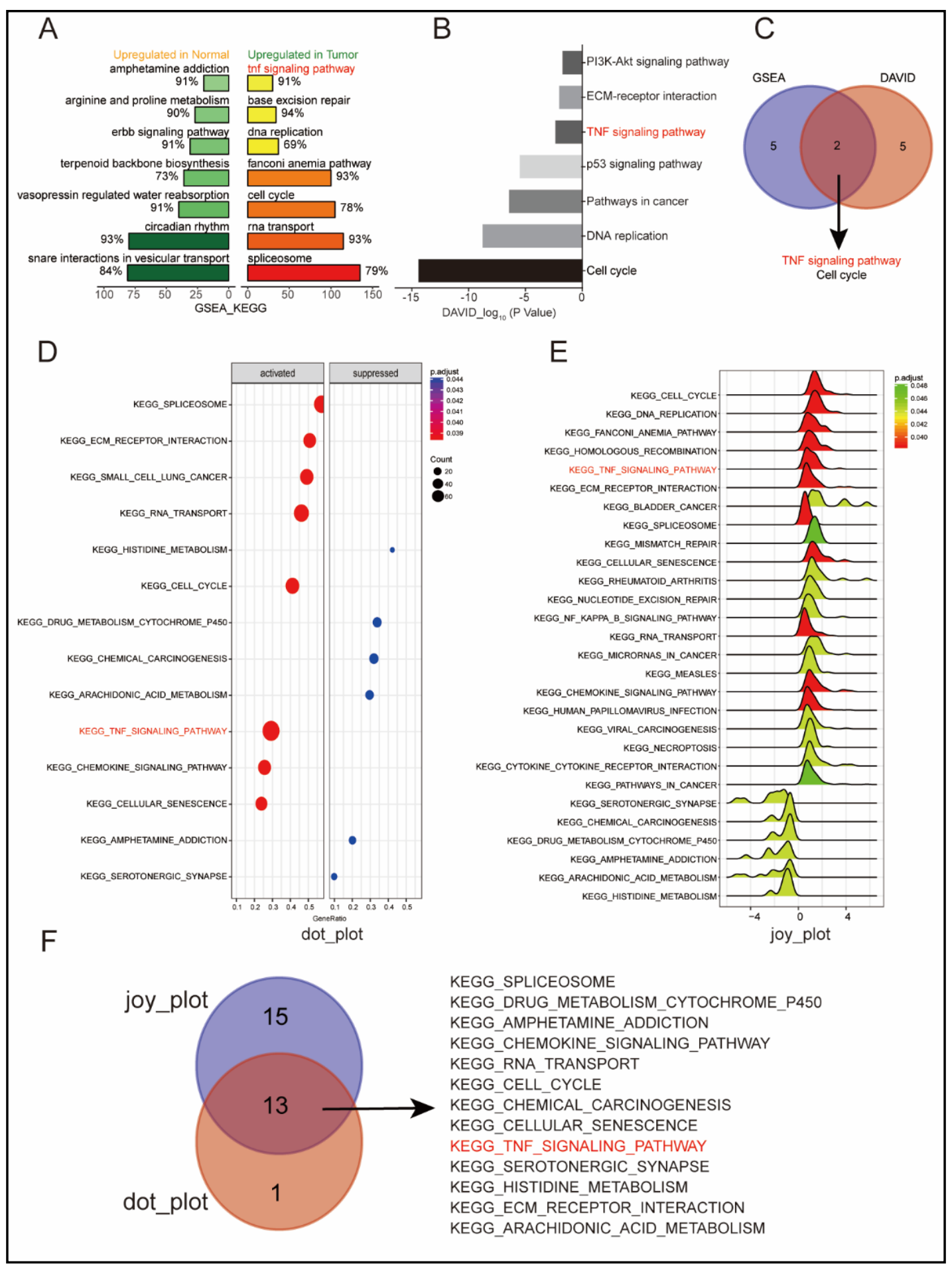

Fig. 3. The TNF signaling pathway that activated in tumor tissues was the key pathway in progression of the CC. (A) The highest 7 KEGG significant pathways respectively in normal and tumor tissues obtained by GSEA. (B)The highest 7 KEGG pathways obtained by functional annotation analysis in DAVID. (C) The venny diagram which showed the common pathways in both GSEA and DAVID analysis. (D) The dot plot and (E) the joy plot which showed the TNF signaling pathway was obviously activated in tumor with $\mathrm{P}<0.05$. $(\mathrm{F}) \mathrm{The}$ venny diagram which showed the TNF signaling pathway was exit in the 13 corporate significantly different pathways revealed by dot plot and joy plot. 


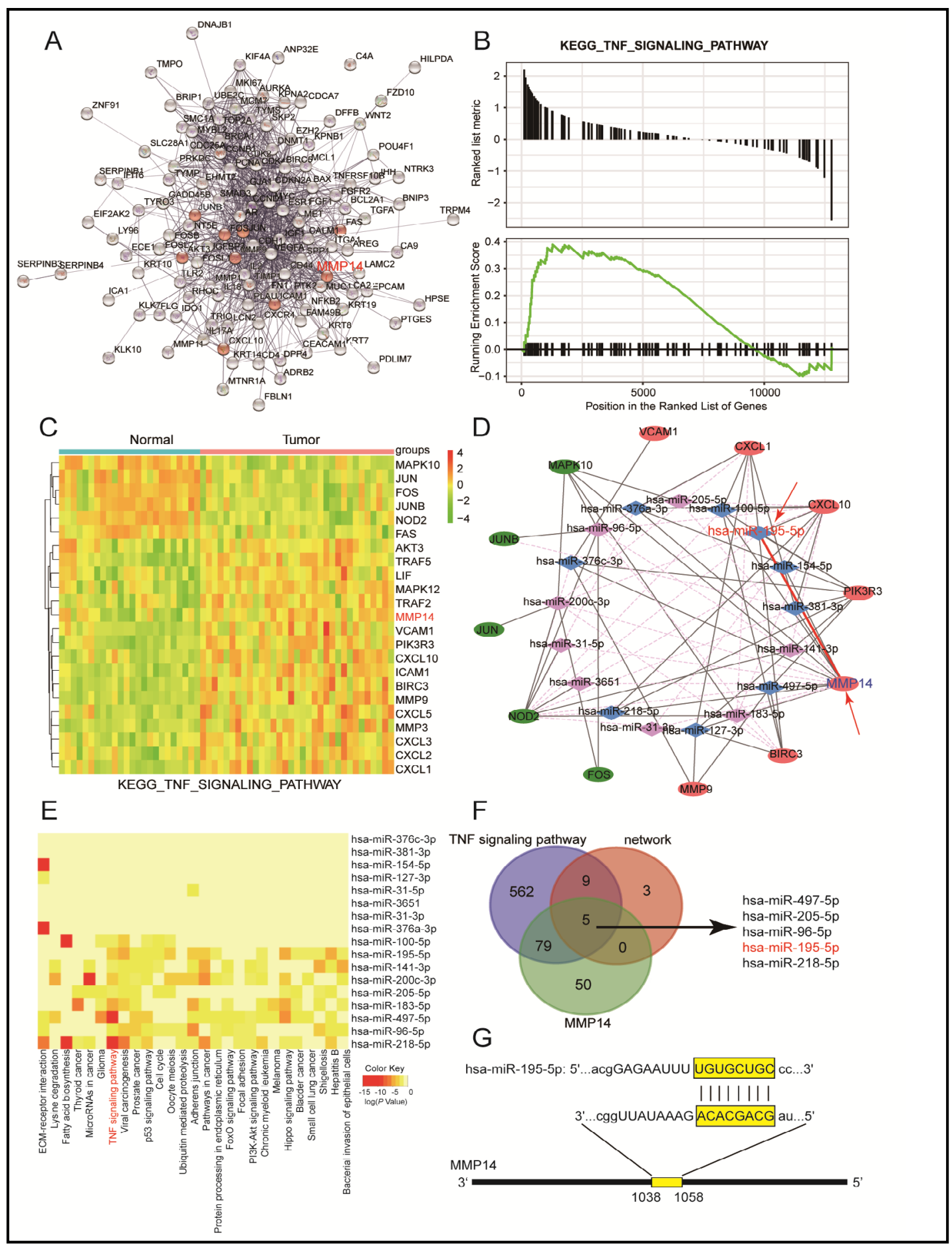

Fig. 4. The miR-195-5p can target the MMP14, and act on the TNF signaling pathway in CC. (A) The interactions of these aberrantly expressed genes in CC and the mRNAs that related to the TNF signaling pathway through string analysis. (B) GSEA showed the TNF signaling pathway was activated in tumors. (C) The heat map detected the differentially expressed mRNAs related to the TNF signaling pathway. (D) The network plot which showed that miR-195-5p was low expression and MMP14 was high expression and the expression of them was negative correlation. (E) The heat map which showed the interaction of the miR195-5p with the TNF signaling pathway obtained by DIANA. (F) The venny diagram showed the common miRNAs of associated TNF signaling pathway, targeting the MMP14 and showed in the network. (G) The binding sites of miR-195-5p on MMP14.

\section{KARGER}




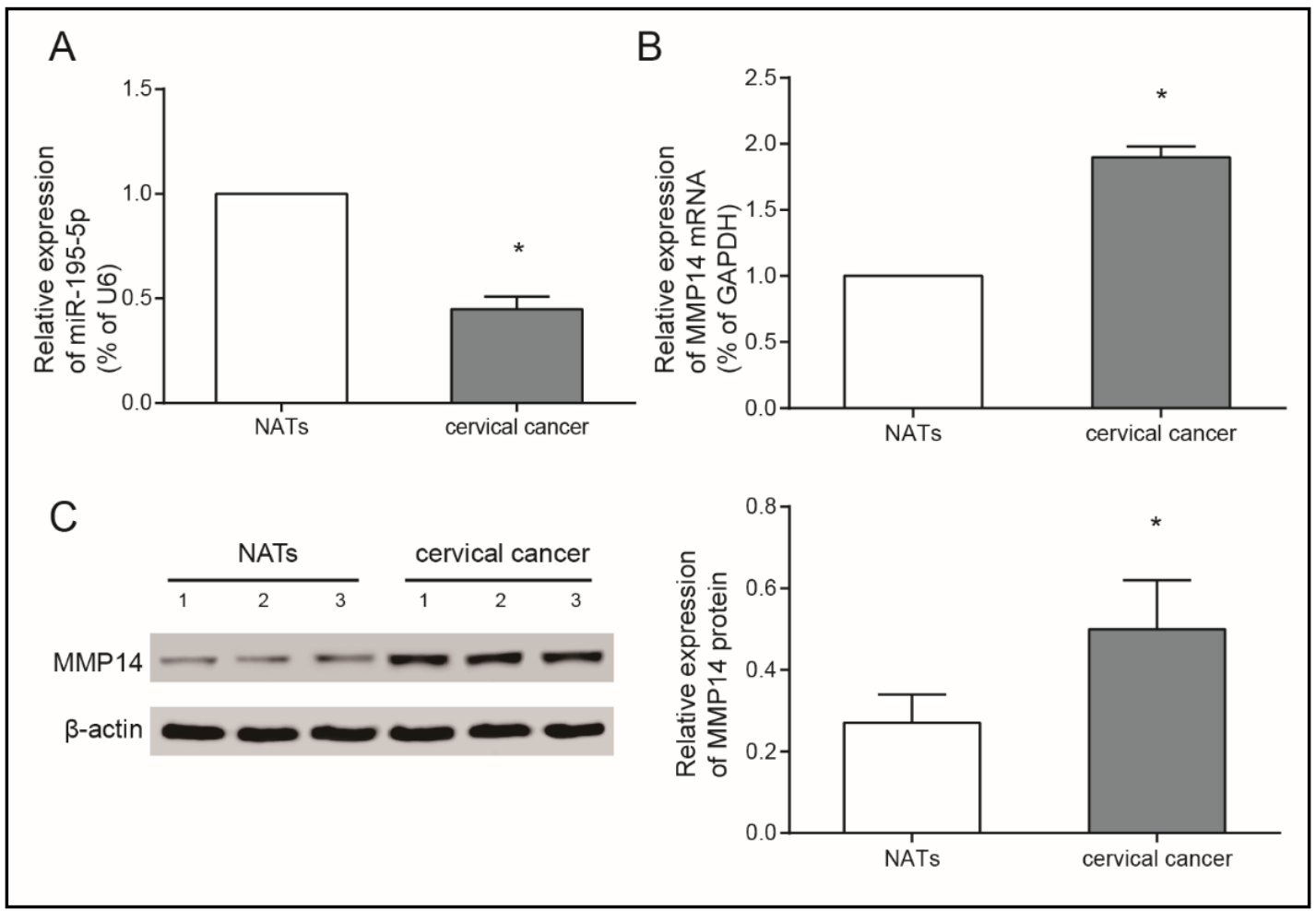

Fig. 5. MiR-195-5p is down-regulated in CC tissues and cells. (A) MiR-195-5p expression was inhibited in CC tissues compared with NATs. The internal control for miR-195 was U6. The data are presented in form of the mean $\pm \mathrm{SD} ; \mathrm{n}=3$; ${ }^{*} \mathrm{P}<0.05$ vs. NATs. (B) RT-PCR result showed that MMP14 expression was promoted in CC tissues compared with NATs. The data are expressed as the mean \pm SD; $n=3 ;{ }^{*} \mathrm{P}<0.05$ vs. NATs. (C) Western blot assay showed that MMP14 expression was increased in CC tissues compared with NATs. The data are expressed as the mean $\pm \mathrm{SD} ; \mathrm{n}=3 ;{ }^{*} \mathrm{P}<0.05$ vs. NATs, non-tumorous adjacent tissue.

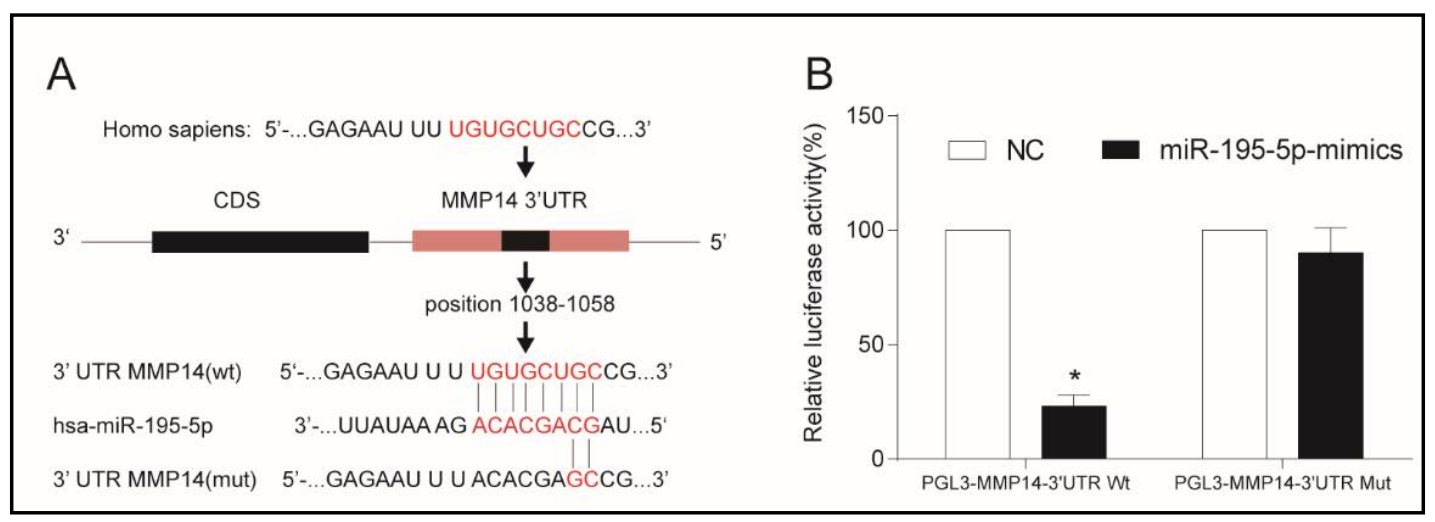

Fig. 6. MMP14 was miR-195-5p's target gene. (A) Sequence alignment of miR-195-5p seed sequence in wild-type (wt) and mutant (mut) MMP14 3'-UTR. (B) Dual-luciferase reporter assay demonstrated that miR-195 repressed the luciferase activity of pGL3-HDGF-3'-UTR Wt but not pGL3-HDGF-3'-UTR Mut in HeLa cells. * means $\mathrm{P}<0.05$, compared to the $\mathrm{NC}$ group. 


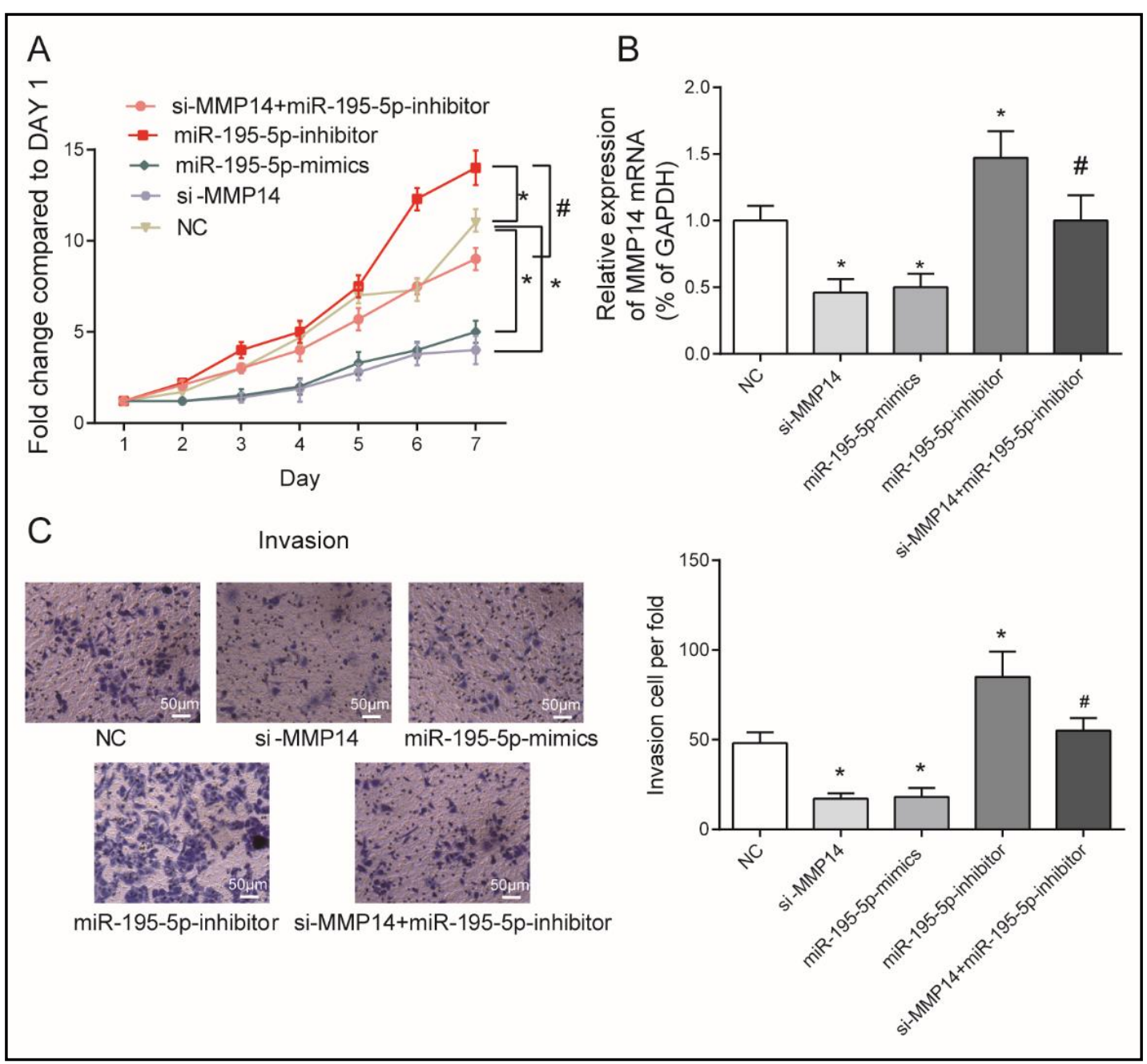

Fig. 7. MiR-195-5p influenced proliferation and invasion of the CC cells by regulating MMP14. (A) CCK-8 assay detected the proliferation ability of 5 TE-1 cell groups. The cells in miR-195-5p-mimics group and siMMP14 group presented the similar cell proliferation ability and were weaker than 3 other groups, while NC group and miR-195-5p-inhibitor+si-MMP14 group were in the second place. MiR-195-5p-inhibitor group had the maximal cell proliferation ability. (B) The level of MMP14 mRNA in the five groups detected by RT-PCR. (C) Transwell migration and invasion assay detected that miR-195-5p-mimics group and si-MMP14 group had minimal migrant and invasive cells. NC group and miR-195-5p-inhibitor+si-MMP14 group had similar migrant and invasive cells. MiR-195-5p-inhibitor group had the most migrant and invasive cells $(200 \times)$.

mimics group compared with NC and si-MMP14 + miR-195-5p-inhibitor group (Fig. 7B). Transwell invasion assay showed that si-MMP14 group and miR-195-5p-mimics group had significantly less cells compared with other groups $(P<0.05)$, while the number of cells in miR-195-5p-inhibitor group was the largest $(P<0.05)$. The difference between NC group and si-MMP14+miR-195-5p-inhibitor group was not significantly different $(P>0.05$, Fig. 7C). The expression of MMP14 in si-TNF- $\alpha$ group was lower than that in NC group (Fig. 8A). Western blot showed that the expression of proteins pathway associated with TNF signal pathway (TNF- $\alpha$, TNFR1 and FAS) was the maximum in miR-195-5p-inhibitor group, the minimum in si-MMP14 and miR-195-5p-mimics groups. The level of proteins associated with TNF signal pathway in NC and si-MMP14 + miR-195-5p-inhibitor groups which had no statistical differences took the second place (Fig. 8B). 


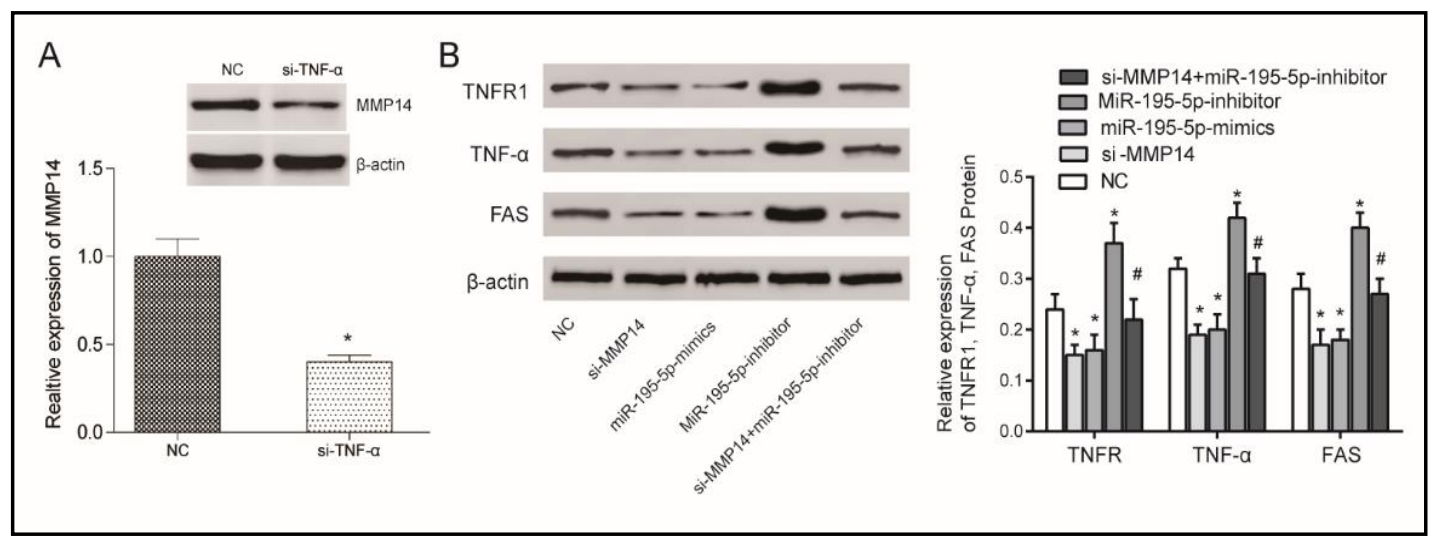

Fig. 8. MiR-195-5p had a positive feedback effect on TNF signaling pathway. (A) Western blot showed the expression of MMP14 was lower after transfected with si-TNFR1 or si-TNF- $\alpha$. ${ }^{*}$ means $\mathrm{P}<0.05$, compared to the NC group. (B) The result of western blot which showed the level of proteins associated with TNF signal pathway. * means $\mathrm{P}<0.05$, compared to NC group; \# means $\mathrm{P}<0.05$, compared to miR-195-5p-inhibitor group.

MiR-195a-5p influenced proliferation of the cervical carcinoma cells by regulating MMP14 in vivo

The volume and weight of the tumors in nude mice were measured after transfected with NC, miR-195-5p-inhibitor and miR-195-5p-mimics respectively. The result presented maximal tumor volume and weight in miR-195-5p-inhibitor group and minimal tumor volume and weight in miR-195-5p-mimics group, while the data in NC group were in the middle $(P<0.05$, Fig. 9A). QRT-PCR result displayed a maximal miR-195-5p expression level and a minimal MMP14 expression level in miR-195-5p-mimics group, while in miR-195-5pinhibitor group, a minimal miR-195-5p expression level and a maximal MMP14 expression level were present (Fig. 9B). Furthermore, western blot revealed that the proteins related to TNF signaling pathway were up-regulated in miR-195-5p-inhibitor group and underexpressed in miR-195-5p-mimics group compared with NC group $(P<0.05)$ (Fig. 9C). All the results above suggested that miR-195-5p interacted with MMP14 and TNF signaling pathway and they might play a role of importance in the evolution of cervical carcinoma. 


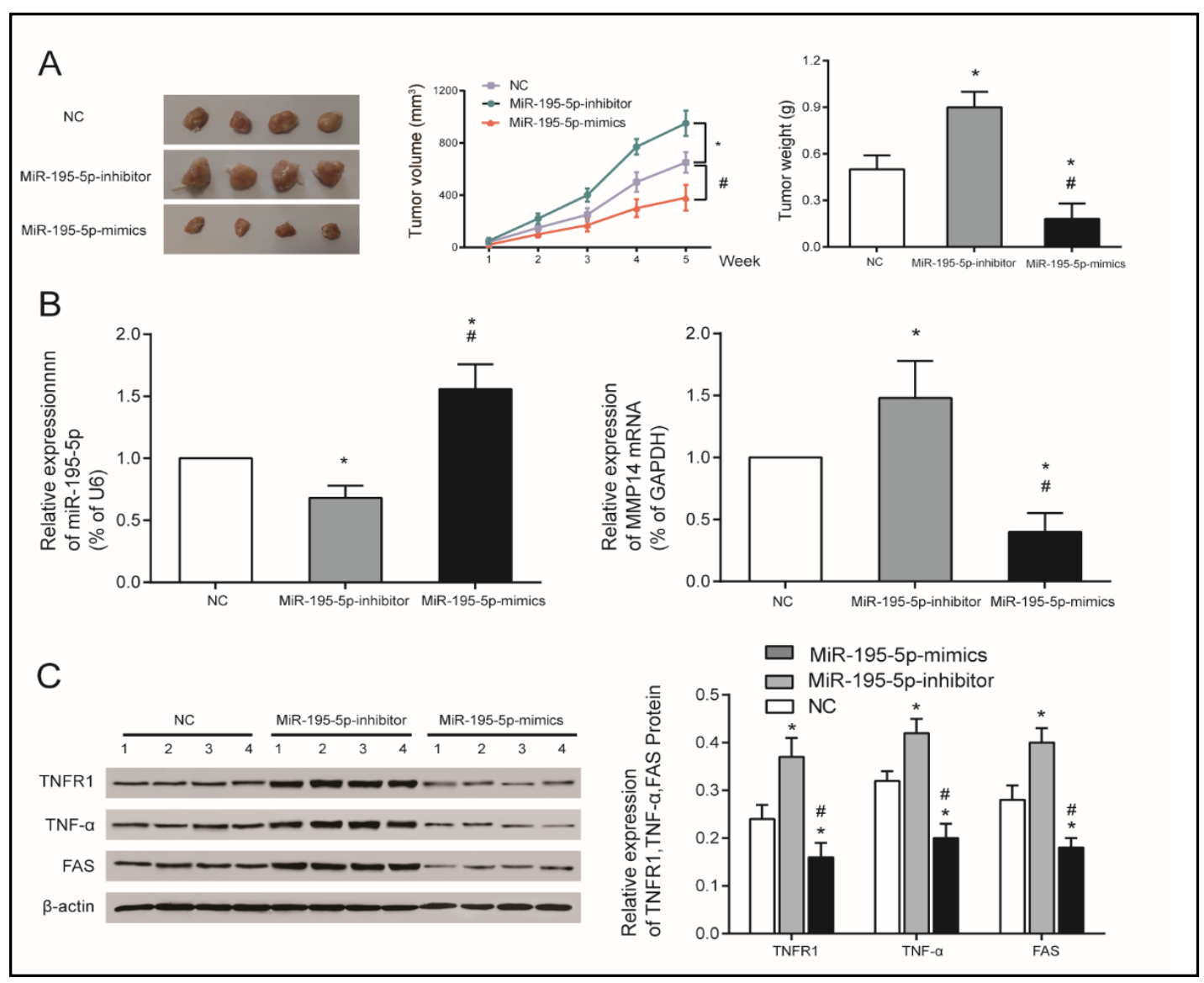

Fig. 9. MiR-195a-5p influenced proliferation of the CC cells by regulating MMP14 in vivo. (A) Images of nude mice tumor specimen. The statistical results of tumor volume showed that, the tumor in miR-195$5 p$-inhibitor group grew fastest and the final volume was maximal, while the tumor in miR-195-5p-mimics group grew slowest and the final volume was minimal. * means $\mathrm{P}<0.05$, compared to NC group. \# means $\mathrm{P}<0.05$, compared to NC group. The statistical results of nude mouse tumor weight showed that, the tumor in miR-195-5p-inhibitor group was heaviest while the tumor in miR-195-5p-mimics group was lightest. * means $\mathrm{P}<0.05$, compared to NC group. \# means $\mathrm{P}<0.05$, compared to miR-195-5p-inhibitor group. (B) The expression level of miR-195-5p and MMP14 were detected by RT-PCR, MiR-195-5p-inhibitor group had the minimal miR-195-5p, maximal MMP14 while miR-195-5p-mimics group had the maximal miR195-5p, minimal MMP14. (C) The levels of proteins related to the TNF signaling pathway in tumor tissue detected by western blot which showed the proteins over-expressed in miR-195-5p-inhibitor group and under-expressed in miR-195-5p-mimics group. * means $\mathrm{P}<0.05$, compared to NC group; \# means $\mathrm{P}<0.05$, compared to miR-195-5p-inhibitor group.

\section{Discussion}

Cervical carcinoma (CC) is the third most prevalent cancer and the fourth mortality leading cancer in females worldwide, for which the treatment remains a problem [3]. The aim of the present study was to find new biomarkers and hereby improve the current therapy methods for CC. We explored the expression condition of miR-195-5p and MMP14 in CC tissues and cells, and discovered that miR-195-5p expression level was decreased while MMP14 was up-regulated in CC tissues and cells. The expression level of proteins related to TNF signaling pathway was also determined and the results showed that it was activated in tissues and cells. Taken together, our study confirmed a targeting relationship between miR195-5p and MMP14 and their function in CC cells by modulating TNF pathway. 


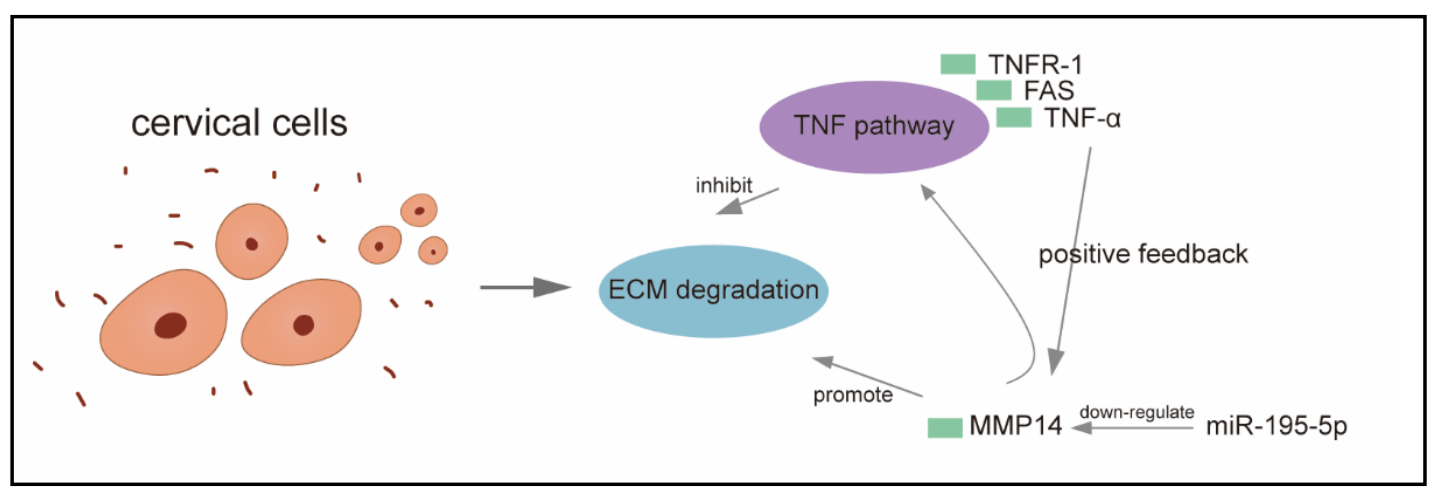

Fig. 10. The molecular mechanism map in CC cell.

In previous studies, miR-195 has been found down-regulated significantly in CC tissues and cells $[2,6]$. For example, Zhang et al. have reported miR-195 as a novel biomarker for detection of CC [5]. In line with these reports, our research displayed the same results based on bioinformatics analysis and in vitro experiments. Inversely, MMP14, believed to be a tumor promoter, was found overexpressed in CC tissues compared with NATs. Although the function of MMP14 in CC has been rarely studied, our results meet the conclusion of Timoshenko et al.'s study, in which MMP14 expression was reported increased in CC cells and its overexpression was found to contribute to the CC tumorigenesis in squamous cell cervical carcinomas [17].

As the most commonly studied member of MMP superfamily, MMP14 has been thoroughly explored, and plentiful researchers put their attention on the link of miRNAs and MMP14. They have reported that a number of miRNAs suppress cancer progress by inhibiting MMP14, such as miR-34a in tongue squamous cell carcinoma, miR-150-5p in hepatoma, miR-181a-5p in human breast and colon cancers and miRNA-337-3p in neuroblastoma [18-21]. However, to the best of our knowledge, our study is the first time the link between miR-195-5p and MMP14 is probed in CC. Surprisingly, similar mechanism was observed in this study. MMP14 was confirmed to be directly targeted by miR-195-5p in CC cells according to the result of bioinformatics analysis and our in vitro experiments, which displayed an antagonism between miR-195-5p and MMP14. Our cell experiments and xenograft experiments both revealed that the proteins associated with TNF signaling pathway were repressed by miR195-5p in CC cells.

Further, it was found out that miR-195-5p could regulate TNF signaling pathway by targeting MMP14. Downregulated MMP14 inhibited the expression of TNF- $\alpha$, TNFR1 and FAS. Knocking down TNF- $\alpha$ declined the expression of MMP14. Hence, we draw a hypothesis that there may exist positive feedback between MMP14 and TNF- $\alpha$. Therefore, MMP14 down-regulated the expression of TNF- $\alpha$, while TNF- $\alpha$ also down-regulated the expression of MMP14. The reason might be that TNF- $\alpha$ increased extracellular matrix (ECM)-related protein [22] and MMP14 was an ECM degrading enzymes [23]. TNF- $\alpha$ down-regulated the expression of MMP14 to promote ECM while MMP14 down-regulated the expression of TNF- $\alpha$ to inhibit ECM. The mechanism map was shown in Fig. 10.

The function of miR-195 in CC has been widely probed, and it is certified that miR-195 inhibits CC cell proliferation, invasion and migration by targeting related genes, while the knockdown of miR-195 has the opposite impact on cell processes [3, 6, 24, 25]. In this study, we further demonstrated that miR-195-5p could lessen CC cell proliferation and invasion ability via targeting MMP14. MMP14 positive expression is considered to connect with a high recurrence of CC patients [10]. And MMP14 down-regulation was found to impair the proliferation and invasion ability of CC cells [26]. Correspondingly, the CCK-8 assay and transwell assay in our study indicated that MMP14 inhibition by miR-195-5p led to a poor proliferation and invasion profile of CC cells. 


\section{Cellular Physiology Cell Physiol Biochem 2018;50:1398-1413 \begin{tabular}{ll|l} 
and Biochemistry Published onlıne: 25 October 2018 & $\begin{array}{l}\text { (c) } 2018 \text { The Author(s). Published by S. Karger AG, Basel } \\
\text { www.karger.com/cpb }\end{array}$ \\
\hline
\end{tabular}}

Li et al.: miR-195-5p/MMP14 Suppresses the Progression of Cervical Carcinoma

\section{Conclusion}

In conclusion, we demonstrated that miR-195-5p could inhibit MMP14 expression to suppress CC development by inactivating TNF signaling pathway. The study might provide miR-195-5p and MMP14 as novel biomarkers in the treatment for CC. In the future studies, more specific function of miR-195-5p and MMP14 in CC cells need to be clarified, such as their role in cell apoptosis and cell cycle.

\section{Acknowledgements}

My deepest gratitude goes to all authors, for their constant encouragement and guidance. They have walked me through all the stages of the writing of this thesis. Without their consistent and illuminating instruction, this thesis could not have reached its present form. Last my thanks would go to my beloved family for their loving considerations and great confidence in me all through these years. This work was supported by the National Natural Science Foundation Youth Project, Mechanism of Cancer-related Fibroblast Cells Activating Stat3/notch Signaling Pathway through IL-6 to Promote Cervical Cancer Metastasis, Radiotherapy and Chemotherapy Resistance (No. 81402153).

\section{Disclosure Statement}

The authors declare no potential conflicts of interest.

\section{References}

1 Xu L, Xu Q Li X, Zhang X: MicroRNA-21 regulates the proliferation and apoptosis of cervical cancer cells via tumor necrosis factor-alpha. Mol Med Rep 2017;16:4659-4663.

2 Song R, Cong L, Ni G, Chen M, Sun H, Sun Y, Chen M: MicroRNA-195 inhibits the behavior of cervical cancer tumors by directly targeting HDGF. Oncol Lett 2017;14:767-775.

3 Du X, Lin LI, Zhang L, Jiang J: microRNA-195 inhibits the proliferation, migration and invasion of cervical cancer cells via the inhibition of CCND2 and MYB expression. Oncol Lett 2015;10:2639-2643.

4 Nakamura H, Taguchi A, Kawana K, Kawata A, Yoshida M, Fujimoto A, Ogishima J, Sato M, Inoue T, Nishida H, Furuya H, Tomio K, Eguchi S, Mori-Uchino M, Yamashita A, Adachi K, Arimoto T, Wada-Hiraike O, Oda K, Nagamatsu T, Osuga Y, Fujii T: STAT3 activity regulates sensitivity to tumor necrosis factor-related apoptosis-inducing ligand-induced apoptosis in cervical cancer cells. Int J Oncol 2016;49:2155-2162.

-5 Zhang Y, Zhang D, Wang F, Xu D, Guo Y, Cui W: Serum miRNAs panel (miR-16-2*, miR-195, miR-2861, miR497) as novel non-invasive biomarkers for detection of cervical cancer. Sci Rep 2015;5:17942.

-6 Zhou Q Han LR, Zhou YX, Li Y: MiR-195 Suppresses Cervical Cancer Migration and Invasion Through Targeting Smad3. Int J Gynecol Cancer 2016;26:817-824.

7 Lin Y, Wang J, Jin W, Wang L, Li H, Ma L, Li Q, Pang T: NHE1 mediates migration and invasion of HeLa cells via regulating the expression and localization of MT1-MMP. Cell Biochem Funct 2012;30:41-46.

$>8$ Tee YT, Liu YF, Chang JT, Yang SF, Chen SC, Han CP, Wang PH, Liao CL: Single-nucleotide polymorphisms and haplotypes of membrane type 1-matrix metalloproteinase in susceptibility and clinical significance of squamous cell neoplasia of uterine cervix in Taiwan women. Reprod Sci 2012;19:932-938.

-9 Liu T, Zhang X, Gao S, Jing F, Yang Y, Du L, Zheng G, Li P, Li C, Wang C: Exosomal long noncoding RNA CRNDE-h as a novel serum-based biomarker for diagnosis and prognosis of colorectal cancer. Oncotarget 2016;7:85551-85563.

10 Wang H, Zhang X, Huang L, Li J, Qu S, Pan F: Matrix metalloproteinase-14 expression and its prognostic value in cervical carcinoma. Cell Biochem Biophys 2014;70:729-734. 


\section{Cellular Physiology Cell Physiol Biochem 2018;50:1398-1413 \begin{tabular}{ll|l} 
DOI: 10.1159/000494602 & $\begin{array}{l}\text { O 2018 The Author(s). Published by S. Karger AG, Basel } \\
\text { www.karger.com/cpb }\end{array}$ \\
\hline
\end{tabular}}

Li et al.: miR-195-5p/MMP14 Suppresses the Progression of Cervical Carcinoma

11 Nakayama I, Shibazaki M, Yashima-Abo A, Miura F, Sugiyama T, Masuda T, Maesawa C: Loss of HoXD10 expression induced by upregulation of miR-10b accelerates the migration and invasion activities of ovarian cancer cells. Int J Oncol 2013;43:63-71.

12 Holdbrooks AT, Britain CM, Bellis SL: ST6Gal-I sialyltransferase promotes tumor necrosis factor (TNF)mediated cancer cell survival via sialylation of the TNF receptor 1 (TNFR1) death receptor. J Biol Chem 2017;10.1074/jbc.M117.801480.

-13 Kaneko K, Williams RO, Dransfield DT, Nixon AE, Sandison A, Itoh Y: Selective Inhibition of Membrane Type 1 Matrix Metalloproteinase Abrogates Progression of Experimental Inflammatory Arthritis: Synergy With Tumor Necrosis Factor Blockade. Arthritis Rheumatol 2016;68:521-531.

14 He B, Yan F, Wu C: Overexpressed miR-195 attenuated immune escape of diffuse large B-cell lymphoma by targeting PD-L1. Biomed Pharmacother 2017;98:95-101.

15 Bras JP, Silva AM, Calin GA, Barbosa MA, Santos SG, Almeida MI: miR-195 inhibits macrophages proinflammatory profile and impacts the crosstalk with smooth muscle cells. PLoS One 2017;12:e0188530.

16 Managit C, Sakurai H, Saiki I: Ethanolic extract of Thevetia peruviana flowers enhances TNF-alpha and TRAIL-induced apoptosis of human cervical cancer cells via intrinsic and extrinsic pathways. Oncol Lett 2017;13:2791-2798.

17 Timoshenko OS, Gureeva TA, Kugaevskaia EV, Solov'eva NI: [Membrane type 1 matrix metalloproteinase (MT1-MMP) and the regulators of its activity as invasive factors in squamous cell cervical carcinomas]. Biomed Khim 2014;60:683-688.

18 Xiang X, Mei H, Zhao X, Pu J, Li D, Qu H, Jiao W, Zhao J, Huang K, Zheng L, Tong Q: miRNA-337-3p suppresses neuroblastoma progression by repressing the transcription of matrix metalloproteinase 14. Oncotarget 2015;6:22452-22466.

19 Li Y, Kuscu C, Banach A, Zhang Q, Pulkoski-Gross A, Kim D, Liu J, Roth E, Li E, Shroyer KR, Denoya PI, Zhu X, Chen L, Cao J: miR-181a-5p Inhibits Cancer Cell Migration and Angiogenesis via Downregulation of Matrix Metalloproteinase-14. Cancer Res 2015;75:2674-2685.

-20 Li T, Xie J, Shen C, Cheng D, Shi Y, Wu Z, Zhan Q Deng X, Chen H, Shen B, Peng C, Li H, Zhu Z: miR-150-5p inhibits hepatoma cell migration and invasion by targeting MMP14. PLoS One 2014;9:e115577.

-21 Jia LF, Wei SB, Mitchelson K, Gao Y, Zheng YF, Meng Z, Gan YH, Yu GY: miR-34a inhibits migration and invasion of tongue squamous cell carcinoma via targeting MMP9 and MMP14. PLoS One 2014;9:e108435.

22 Watanabe Y, Osaki H, Akaike T: TNF-alpha bifunctionally induces proliferation in primary hepatocytes: role of cell anchorage and spreading. J Immunol 1997;159:4840-4847.

-23 Nakajima K, Tazawa I, Yaoita Y: Thyroid Hormone Receptor alpha- and beta-Knockout Xenopus tropicalis Tadpoles Reveal Subtype-Specific Roles During Development. Endocrinology 2018;159:733-743.

24 Li Z, Wang H, Wang Z, Cai H: MiR-195 inhibits the proliferation of human cervical cancer cells by directly targeting cyclin D1. Tumour Biol 2016;37:6457-6463.

25 Wang N, Wei H, Yin D, Lu Y, Zhang Y, Zhang Q Ma X, Zhang S: MicroRNA-195 inhibits proliferation of cervical cancer cells by targeting cyclin D1a. Tumour Biol 2016;37:4711-4720.

-26 Zhang YH, Wang JJ, Li M, Zheng HX, Xu L, Chen YG: Matrix Metallopeptidase 14 Plays an Important Role in Regulating Tumorigenic Gene Expression and Invasion Ability of HeLa Cells. Int J Gynecol Cancer 2016;26:600-606. 